Check for updates

Cite this: J. Mater. Chem. C, 2021 9, 925

Received 9th September 2020, Accepted 10th November 2020

DOI: $10.1039 / \mathrm{d0tc04309e}$

rsc.li/materials-c

\title{
Precision tuning of rare-earth-doped upconversion nanoparticles via droplet-based microfluidic screening $\dagger$
}

\author{
Shangkun Li, (D) a Yingchao Meng, (D) a Yujia Guo, ${ }^{a}$ Tian Liu, ${ }^{b}$ Stavros Stavrakis, (D) ${ }^{a}$ \\ Philip D. Howes (D) *a and Andrew J. deMello (D) *a
}

\begin{abstract}
Rare-earth-doped upconversion nanoparticles (UCNPs) show great promise in a range of applications, including biological imaging and sensing, solar cells and security inks. Although their emission color can be tuned widely as a function of host matrix and dopant composition, the high dimensionality of the associated parameter space and the sensitivity of emission to these parameters make optimization and precision tuning difficult. Herein, we present a new time-efficient synthetic route to NaYF $4: Y_{b}, E_{\text {, }} \mathrm{Tm}$ UCNPs and a high-throughput microfluidic reactor to synthesize and precisely tune the emission characteristics of the particles in situ and in real time. We synthesize a range of particles with optimized emission intensity and wide color distribution by changing the doping degree of sensitizer $\mathrm{Yb}$ to provide for green-orange tunability, and the ratio of Tm-Er to give green-blue tunability. With the two tunable dimensions, we realize true white light emitting UCNPs based on optimized red, green and blue (RGB) emission ratios from a single composition $\mathrm{NaYF}_{4}: \mathrm{Yb}, \mathrm{Er}, \mathrm{Tm}$ nanocrystal-a demanding task for such materials-with CIE 1931 coordinates of $(0.29,0.34)$ and doping degrees of $60 \% \mathrm{Yb}, 0.45 \% \mathrm{Er}$, and $1.05 \%$ $\mathrm{Tm}$. Finally, we demonstrate the efficacy of these materials in a thin film format through the fabrication of an anti-counterfeit device.
\end{abstract}

\section{Introduction}

White light-emitting materials have attracted enormous attention for the development of energy efficient and high performance lighting and displays. ${ }^{1-3}$ Materials of interest include polymers, small molecules, quantum dots and rare-earth-doped upconversion nanoparticles (UCNPs). ${ }^{4-8}$ Whilst white light emission is relatively easy to achieve by mixing different colors of emitter in a heterogeneous system, such an approach suffers from problems associated with phase separation of the different emitting bodies, unwanted energy transfer, low stability and/ or the need for multiple excitation wavelengths. ${ }^{9,10}$ Accordingly, new materials that can emit red-green-blue (RGB) colors from a single entity are highly sought after.

Rare-earth (RE) UCNPs, typically co-doped with sensitizer ions of ytterbium $\left(\mathrm{Yb}^{3+}\right)$ and activator ions of $\mathrm{X}^{3+}(\mathrm{X}=\mathrm{Er}, \mathrm{Tm}$, and/or Ho) in the same nanocrystal host, can yield non-linear

\footnotetext{
${ }^{a}$ Institute for Chemical and Bioengineering, Department of Chemistry and Applied Biosciences, ETH Zürich, Vladimir Prelog Weg 1, 8093 Zürich, Switzerland. E-mail: andrew.demello@chem.ethz.ch,philip.howes@chem.ethz.ch

${ }^{b}$ Laboratory for Multifunctional Materials, Department of Materials, ETH Zürich, Vladimir Prelog Weg 1, 8093 Zürich, Switzerland

$\dagger$ Electronic supplementary information (ESI) available. See DOI: 10.1039/ dotc04309e
}

anti-Stokes luminescence via sequential absorption of two or more low energy near-infrared (NIR) photons. ${ }^{11,12}$ UCNPs are free of reabsorption, emit with narrow peaks and are photostable, with tunable lifetimes and low toxicity. ${ }^{13,14}$ Longwavelength NIR excitation leads to less scatter, minimizes photodamage and autofluorescence of biological samples and allows deep tissue penetration. ${ }^{15}$ Standout applications of UCNPs include biological imaging and therapy, ${ }^{16}$ photonic devices, ${ }^{17}$ luminescence thermometry, ${ }^{18}$ biosensing, ${ }^{19}$ and anti-counterfeiting. ${ }^{20-22}$ Regarding white light emission, it is possible to tune rare-earth UCNPs to act as single entity white light emitters, overcoming the previously noted problems encountered in heterogeneous populations. For example, Sivakumar et al. demonstrated a white light thin film made from $\mathrm{RE}^{3+}$-doped $\mathrm{LaF}_{3}$ nanoparticles, which could be excited using a single NIR source. ${ }^{4}$ Subsequently, Wang et al. reported white upconversion rare-earth oxide materials $\mathrm{Tm}_{2} \mathrm{O}_{3}$ and $\mathrm{Yb}_{2} \mathrm{O}_{3}$, which achieved pure white light emission using single source excitation. ${ }^{23}$ More recently, Zhang et al. designed a core-shellshell nanostructure incorporating several $\mathrm{RE}^{3+}$ within a single nanocrystal, and whose emission colors could be tuned by changing the excitation power density for white light. ${ }^{9}$ Addi-

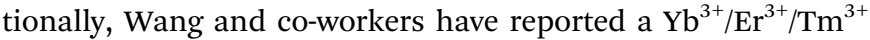
tridoped hexagonal $\beta-\mathrm{NaYF}_{4}$ microrod for white-light lasing at 
room temperature. ${ }^{24}$ Despite these successes, the precision tuning of emission characteristics across a wide range and using a single nanocrystal composition is still a challenge, and there is much potential still to be unlocked.

During the last decade, several UCNP synthesis methods have been proposed, ${ }^{25}$ including hydrothermal, thermal decomposition, and thermal co-precipitation reactions. ${ }^{26-28}$ However, these typically require harsh reaction conditions (i.e. high temperatures and/or high pressures), and extended reaction times. Moreover, bulk reaction approaches to nanocrystal synthesis typically necessitate large investments of time and materials to effectively map an inherently complex reaction parameter space. This is especially problematic for UCNPs, as their properties are extremely sensitive to the exact preparation conditions, in particular the doping degree of the sensitizers and activators, and thus they would benefit hugely from a more comprehensive characterization and optimization process. Further, we propose that the development of UCNPs will drastically benefit from a more efficient, economic and greener strategy for fast parameter screening, with a view to optimizing properties for advanced applications.

Since the pioneering works of nearly two decades ago, ${ }^{29-31}$ great strides have been made in establishing the field of microfluidic-based nanoparticle synthesis. In the field of rareearth UCNPs, previous reports have demonstrated the utility of continuous flow microfluidic approaches. ${ }^{32-34}$ However, such methods are not suitable for high-throughput screening because of difficulties in controlling cross-contamination between solutions run with different reaction parameter sets. In contrast, droplet-based microfluidic reactors have engendered a time-efficient high-throughput screening approach for some years now, ${ }^{35}$ due to efficient heat and mass transfer within droplets, and the ability to analyze products and tune reactions in real-time. ${ }^{36}$ However, rare-earth UCNPs have not benefitted from this approach due to their restrictively long reaction times and the difficulty in preparing single compositional element sources (i.e. precursors that deliver only one of the elements that form the final $\mathrm{NC}$ ). Unlike in common bulk reactions where the $\mathrm{RE}^{3+}, \mathrm{Na}^{+}$and $\mathrm{F}^{-}$are premixed in one solution, in the proposed high-throughput screening method it is imperative that each of the compositional elements can be delivered by a separate syringe to allow fine control of reaction stoichiometry. However, this isolation of precursors causes solubility issues, which necessitates redesigning the reaction in terms of solvent, reagents and ligands.

In this paper, we detail a new synthetic approach for rareearth UCNPs that allows for facile parametric space mapping and fine tuning of RGB emission under NIR excitation. Focussing on $\mathrm{Yb}-\mathrm{Er}-\mathrm{Tm}$ tri-doped $\mathrm{NaYF}_{4}$ UCNPs, we first develop a novel reaction formulation that enables time-efficient screening, where each compositional element can be delivered in a separate precursor for precise tuning of elemental ratios (in terms of $\mathrm{F}^{-}$and $\mathrm{Na}^{+}$for the host matrix, $\mathrm{Yb}^{3+}$ as a sensitizer, and $\mathrm{Er}^{3+}$ and $\mathrm{Tm}^{3+}$ as the activators). Second, and to achieve rapid and efficient parameter mapping and product optimization, we show for the first time the use of a high-throughput droplet-based microfluidic reactor with inline emission analysis for rapid lanthanide-doped UCNP synthesis and compositional tuning. This approach was used to scan and tune the RGB emission attainable from a single $\mathrm{NaYF}_{4}: \mathrm{Yb}, \mathrm{Er}, \mathrm{Tm}$ nanocrystal composition (as opposed to a mixed population) by optimizing elemental ratios, engendering tunable true white light emission based on optimized RGB intensity ratios under single excitation. These developments encompass a new strategy for rapid UCNP synthesis, characterization and optimization. Expansion of this approach will pave the way for advanced applications of these promising materials.

\section{Results and discussion}

\subsection{Experimental design}

We developed a droplet-based microfluidic platform (see Fig. 1a) for reaction parameter screening of $\mathrm{NaYF}_{4}: \mathrm{Yb}, \mathrm{Er}, \mathrm{Tm}$ UCNPs using integrated real-time inline infrared laser $(980 \mathrm{~nm})$ excitation and spectral reading (see $\mathrm{ESI}_{\dagger} \dagger$ for full details). Briefly, seven syringes were used to deliver the reaction components, with four syringes filled with $\mathrm{Re}^{3+}$ precursors at equal molar concentrations, two syringes with the $\mathrm{Na}^{+}$and $\mathrm{F}^{-}$ precursors, and a final syringe with the carrier oil. The four $\mathrm{Re}^{3+}$ precursors were initially mixed in a seven-port manifold, before being delivered into a second manifold to be mixed with the $\mathrm{Na}^{+}$and $\mathrm{F}^{-}$precursors and the oil to segment the flow into droplets. This arrangement allows precise control of the ratios between the $\mathrm{RE}^{3+}$ regents, as well between the $\mathrm{Na}^{+}$and $\mathrm{RE}^{3+}$, and $\mathrm{RE}^{3+}$ to $\mathrm{F}^{-}$. Formed droplets then travelled through a heating module, where the droplets are conveyed through PTFE tubing around a heated copper rod. The formed UCNPs were monitored in situ with an inline photoluminescence system, requiring a minimum time of 3 minutes to collect data per reaction parameter set.

The ability to rapidly scan precursor ratios allows for fast, accurate, reproducible and efficient parameter screening. ${ }^{37}$ In this regard, it should be noted that droplet-based microfluidic systems benefit from rapid heat and mass transfer, which ensures prompt mixing and temperature equilibration on ultra-short timescales. ${ }^{38}$ To assess the temperature equilibration time in our reactor, we conducted computational fluid dynamic (CFD) simulations of the heat transfer from the heating rod (Fig. 1b) into the reaction solution (Fig. 1c and Fig. S1, ESI $\dagger$ ). At a total flow rate $80 \mu \mathrm{L} \min ^{-1}$, the reaction solution reaches the target temperature (i.e. room temperature to $220{ }^{\circ} \mathrm{C}$ ) after $3 \mathrm{~mm}$ of travel, and within $500 \mathrm{~ms}$ (Fig. 1d). This significantly improves upon the heat transfer efficiency obtained in flask-based systems, and reduces reaction times for UCNPs synthesis. Further details on the simulations are provided in the ESI. $\dagger$

To tune or optimize the properties of $\mathrm{NaYF}_{4}: \mathrm{Yb}, \mathrm{Er}, \mathrm{Tm}$ UCNPs by reaction parameter screening, the precursor ratios between each constituent element must be controllable, meaning that each element must to be delivered in a separate precursor. This is not typically the case in bulk reactions, where 
$\mathbf{a}$
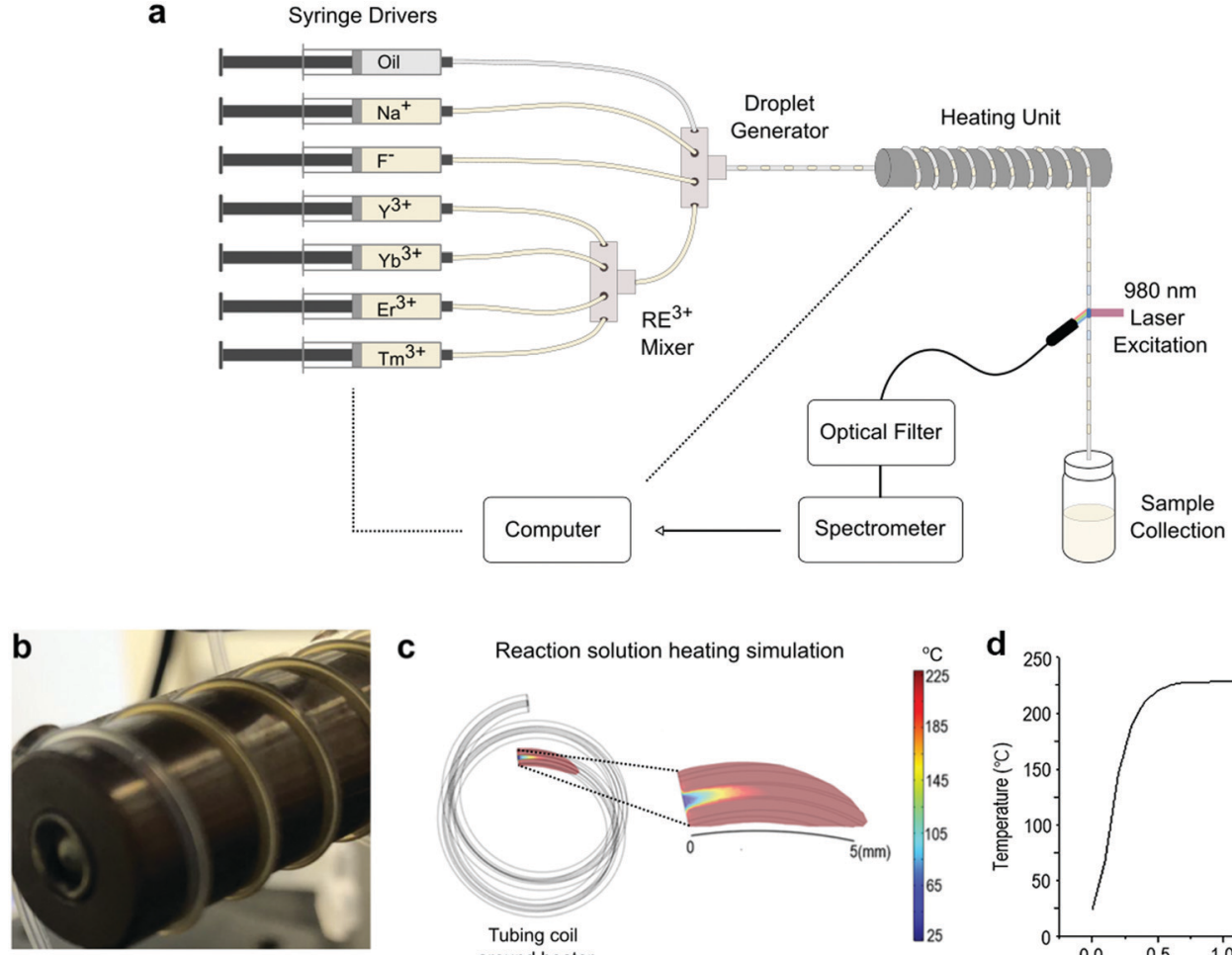

C
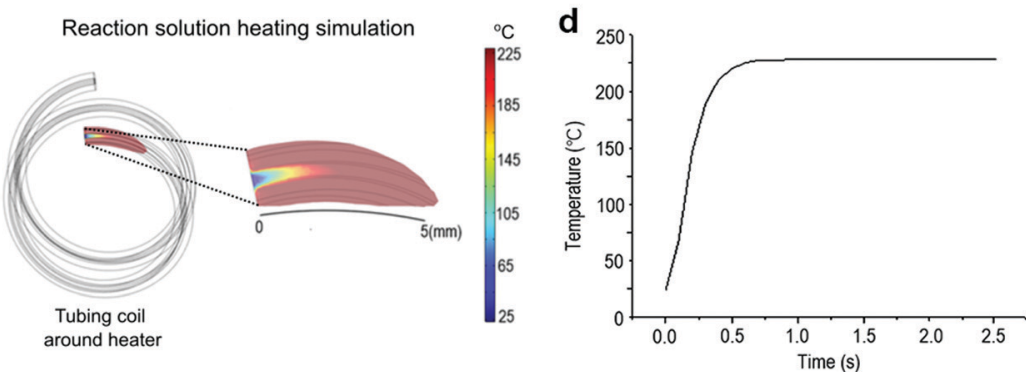

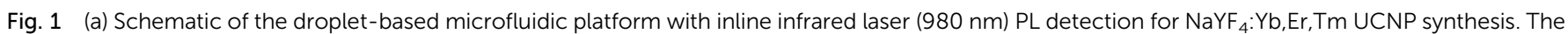
microfluidic platform incorporates syringe pumps, a heating controller, and a real-time fluorescence detection system together. (b) Photograph of the heating loops around a $2 \mathrm{~cm}$ diameter heating rod. (c) Temperature distribution of the flow at the beginning of the heater, with temperatures of the heater and surrounding air at $220^{\circ} \mathrm{C}$ and $25^{\circ} \mathrm{C}$, respectively. (d) Variation of the temperature of the reaction solution as a function of heating time.

$\mathrm{Na}^{+}, \mathrm{RE}^{3+}$, and $\mathrm{F}^{-}$are combined within a single precursor. ${ }^{26-28}$ In our new strategy, we are able to deliver each element in a separate precursor. Furthermore, instead of using 1-octadecene, we employed TEGDME as the solvent for all precursors due to its high boiling point $\left(275{ }^{\circ} \mathrm{C}\right)$ and excellent solubility for $\mathrm{F}^{-}, \mathrm{Na}^{+}$, and $\mathrm{RE}^{3+}$. Finally, it should be noted that modulation of droplet residence times in the heated zone allows for control over the total reaction time.

\subsection{Parameter optimization and color tuning for $\mathrm{NaYF}_{4}: \mathrm{Yb}, \mathrm{Er}$}

In $\mathrm{NaYF}_{4}: \mathrm{Yb}, \mathrm{Er}$ nanocrystals, $\mathrm{NaYF}_{4}$ is the host lattice for lanthanide ions, $\mathrm{Yb}^{3+}$ acts as a sensitizer to absorb the nearinfrared laser light, whilst $\mathrm{Er}^{3+}$ is the activator for green and red emission. Optimization of emission intensity and tuning of spectral profile (i.e. color) can be achieved by varying the precursor ratios for the host lattice, sensitizer and activators, along with the reaction temperature. Accordingly, we assessed the following ratios in this work: $R_{1}=\mathrm{Na} / \mathrm{RE} ; R_{2}=\mathrm{F} / \mathrm{RE} ; R_{3}=\mathrm{Yb} /$ $\mathrm{RE}$ and $R_{4}=\mathrm{Er} / \mathrm{RE} . R_{1}(\mathrm{Na} / \mathrm{RE})$ and $R_{2}(\mathrm{~F} / \mathrm{RE})$, which are the critical ratios for the host lattice $\mathrm{NaYF}_{4}$. While screening these two parameters, we initially set the RE doping to typical values reported in the literature (molar percentages of $80 \% \mathrm{Y}$, $18 \% \mathrm{Yb}$, and $2 \% \mathrm{Er}$ ). ${ }^{33,39}$ As shown in Fig. $2 \mathrm{a}$ and b, the PL intensity of the formed $\mathrm{NaYF}_{4}: \mathrm{Yb}$,Er nanocrystals was relatively weak when $R_{1}$ was lower than 1.2 , but rapidly increased to a maximum at $R_{1}=1.8$.
To better compare the green and red emission from NaY$\mathrm{F}_{4}: \mathrm{Yb}, \mathrm{Er}$, the PL peaks at $652 \mathrm{~nm}\left({ }^{4} \mathrm{~F}_{9 / 2} \rightarrow{ }^{4} \mathrm{I}_{15 / 2}\right)$ and $543 \mathrm{~nm}$ $\left({ }^{4} \mathrm{~S}_{3 / 2} \rightarrow{ }^{4} \mathrm{I}_{15 / 2}\right)$ were chosen as indices (see Fig. $5 \mathrm{c}$ ). The optimized PL intensity occurs when $R_{1}$ lies between 1.5 and 2.5 (peak at 1.8), where both the green and red light obtain their maximum intensity, with the intensity at $652 \mathrm{~nm}$ always being higher than at $543 \mathrm{~nm}$. However, when $R_{1}$ is larger than 2.5, the PL intensity at $543 \mathrm{~nm}$ exceeds the PL intensity at $652 \mathrm{~nm}$. This switching of dominance between the two peaks allows facile tuning of the green to red emission ratio via modulation of the $\mathrm{Na} / \mathrm{RE}$ molar ratio $\left(R_{1}\right)$.

The PL intensity of UCNPs was then optimized using an $R_{2}$ (F/RE) scan, from 2.4 to 6.0, uncovering an optimal range of 3.2-3.6 (Fig. 2c and d). Interestingly this ratio is less than the $4: 1$ stoichiometry of $\mathrm{NaYF}_{4}$. Indeed, the most popular methods for $\mathrm{NaYF}_{4}: \mathrm{Yb}, \mathrm{Er}$ synthesis (Table 1) all use a stoichiometry of 4 or higher.

Ratios $R_{3}(\mathrm{Yb} / \mathrm{RE})$ and $R_{4}(\mathrm{Er} / \mathrm{RE})$ control the rare-earth element doping (i.e. percentage versus total RE content) for $\mathrm{NaYF}_{4}$ :Yb,Er. From the $R_{3}$ scan we observed divergent behaviour for the red and green PL peaks (Fig. 2e and f). The PL from both red and green increases up to $15 \%$ doping, where they both form a local maximum. However, above 15\%, green emission decreases steadily whereas the red emission increases again. This phenomenon means that the red to green intensity ratio may be adjusted via control of $\mathrm{Yb}$ content, $R_{3}$. 

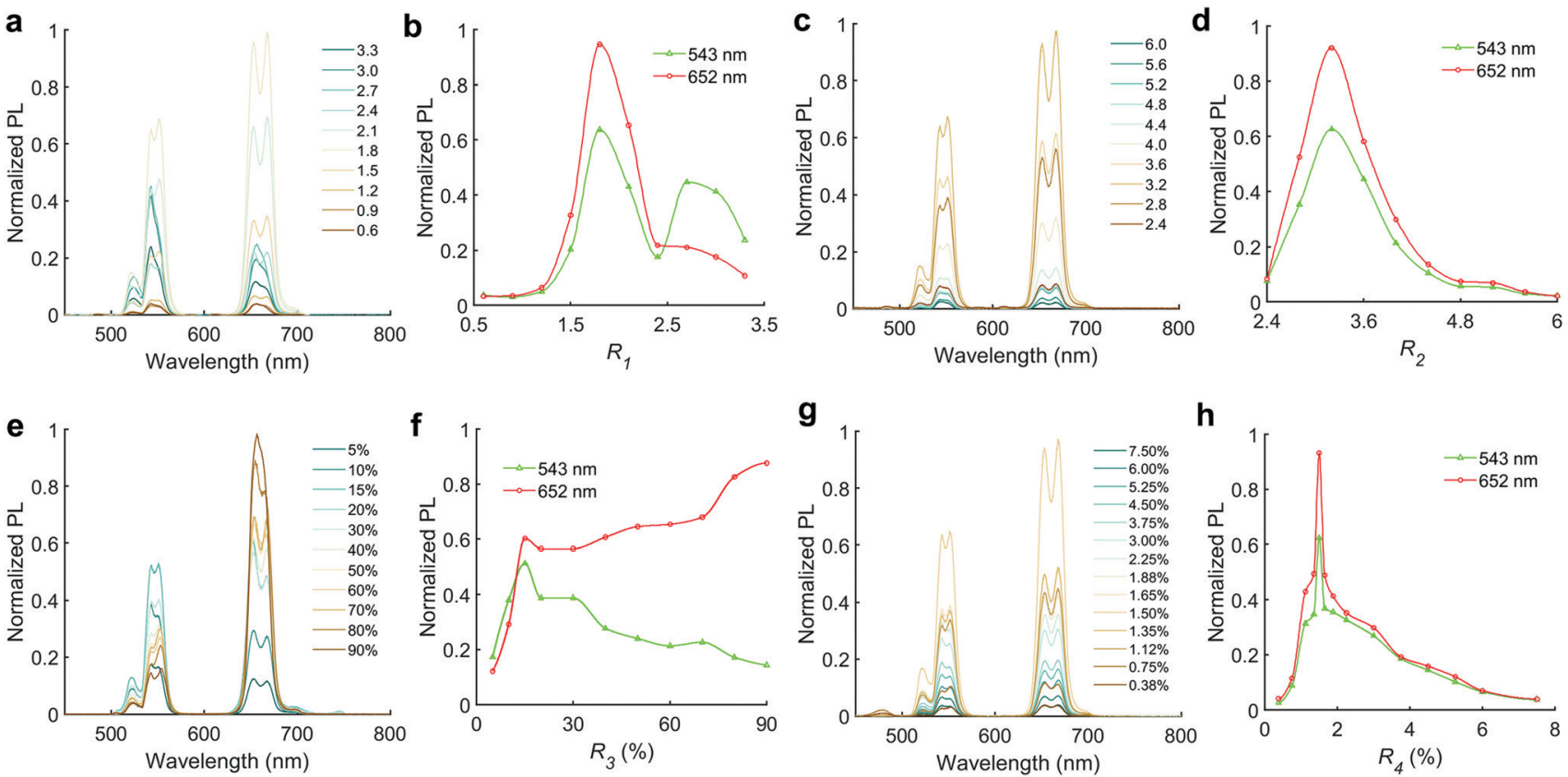

Fig. 2 Optimization of reagent molar ratios $R_{1}-R_{4}$ for $\mathrm{NaYF}_{4}: \mathrm{Yb}, \mathrm{Er}$ nanocrystals. (a and b) Effect of the Na/RE molar ratio $\left(R_{1}\right)$ on PL. (c and d) Effect of the F/RE molar ratio $\left(R_{2}\right)$ on $\mathrm{PL}$. (e and f) Effect of the Yb/RE molar ratio $\left(R_{3}\right)$ on PL. ( $g$ and h) Effect of Er/RE molar ratio $\left(R_{4}\right)$ on the PL of UCNPs. All reactions were performed at $230{ }^{\circ} \mathrm{C}$ for 48 seconds. To index the green and red color, peaks at $543 \mathrm{~nm}$ and $652 \mathrm{~nm}$ were chosen, respectively.

After normalizing the PL at $543 \mathrm{~nm}$ (Fig. 3a), it is evident that the red emission increases with $\mathrm{Yb}^{3+}$ doping concentration (from $5 \%$ to $90 \%$ ) due to the influence of the cross-relaxation process. ${ }^{24,40}$ To achieve higher intensity emission from UCNPs, the activator doping ratio of $\mathrm{Er}^{3+}$ was optimized in Fig. $2 \mathrm{~g}$ and $\mathrm{h}$, where the optimum value of $R_{4}$ was found to be $1.5 \%$.

Fig. 3c presents a CIE chromaticity diagram, where the CIE chromaticity coordinates vary from $x=0.3332, y=0.6503$ (green) to $x=0.5020, y=0.4710$ (orange) by increasing the doping ratio of $\mathrm{Yb}^{3+}$ from 5 to $90 \%$. To obtain a better understanding of color evolution, spectra were converted into RGB tristimulus values (Fig. 3b) based on the CIE colour matching functions. ${ }^{41}$ The RGB percentages are shown in Fig. 3d. With increasing $\mathrm{Yb}^{3+}$ content, the percentage of red emission smoothly increases from 21 to $65 \%$. In contrast, the percentage of green emission decreases from 79 to $34 \%$. The blue emission is near zero across the whole range. Overall, we see that the emission color from $\mathrm{NaYF}_{4}: \mathrm{Yb}$,Er UCNPs can be tuned from green to orange by changing the doping ratio of $\mathrm{Yb}^{3+}$ in $\mathrm{RE}$.

After scanning precursor ratios for emission color tuning, we conducted a study of the impact of reaction temperature and residence time, with a view to further optimizing reaction parameters and understanding the influence of these factors on UCNP properties (Fig. 4). For temperature optimization, we used the optimal ratios established previously, i.e. $R_{1}=1.8, R_{2}=$ $3.2, R_{3}=15 \%$, and $R_{4}=1.5 \%$, respectively, with a total reaction time of 48 seconds. In the range of 160 to $240{ }^{\circ} \mathrm{C}$, there was a continual increase in PL from effectively zero at $160{ }^{\circ} \mathrm{C}$ (Fig. $4 \mathrm{~b}$ ).

Table 1 Reaction parameters of different methods for synthesis of $\mathrm{NaYF}_{4}: \mathrm{Yb}, \mathrm{Er} \cup \mathrm{UNPs}\left(R_{1}=\mathrm{Na} / \mathrm{RE} ; R_{2}=\mathrm{F} / \mathrm{RE} ; R_{3}=\mathrm{Yb} / \mathrm{RE} ; R_{4}=\mathrm{Er} / \mathrm{RE}\right)$

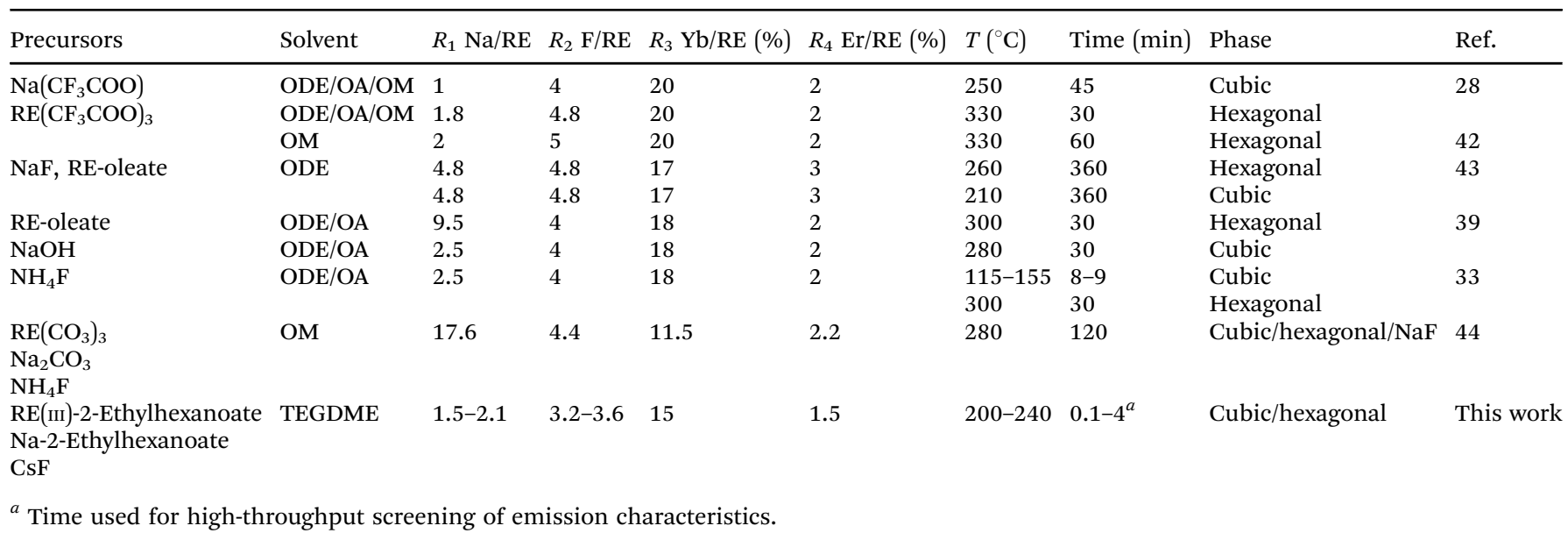



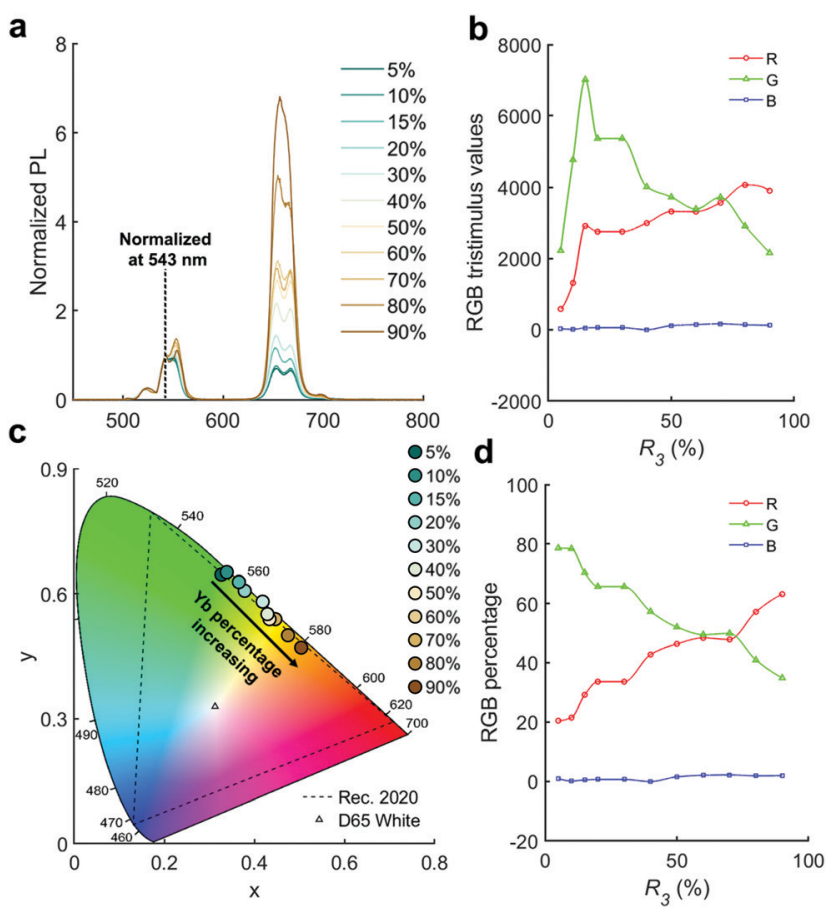

Fig. 3 Emission color of $\mathrm{NaYF}_{4}: \mathrm{Yb}, \mathrm{Er}$ UCNPs was tuned by changing the $\mathrm{Yb}$ doping degree. (a) Normalized spectra of $\mathrm{NaYF}_{4}: \mathrm{Yb}, \mathrm{Er}$ UCNPs with $\mathrm{Yb}$ doping degree $\left(R_{3}\right)$ ranging from $5 \%$ to $90 \%$. (b) Plot of the CIE1931 color coordinates of the NaYF $4: Y b, E r$ UCNPs with different values of $R_{3}$. (c) The $\mathrm{RGB}$ tristimulus values of the $\mathrm{NaYF}_{4}: \mathrm{Yb}, \mathrm{Er}$ with different $\mathrm{Yb}$ molar ratios $\left(R_{3}\right)$. (d) The RGB percentage of UCNPs with different $\mathrm{Yb}$ molar ratios $\left(R_{3}\right)$.

It is evident that the PL has not reached a global maximum by $240{ }^{\circ} \mathrm{C}$, however this was the highest accessible temperature using
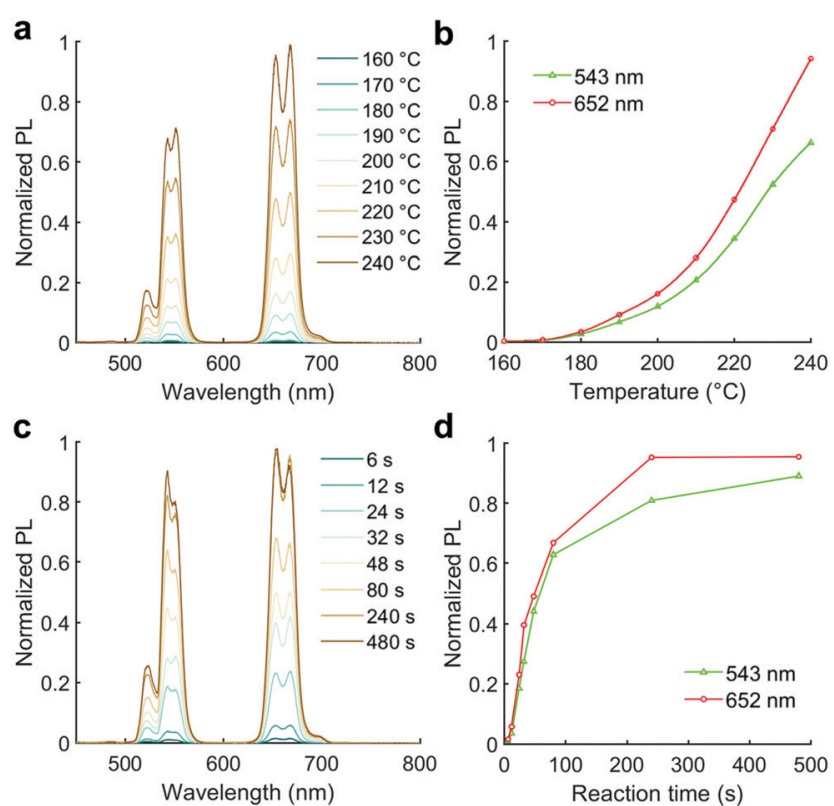

Fig. 4 Temperature and residence time optimization for $\mathrm{NaYF}_{4}: \mathrm{Yb}, \mathrm{Er}$ nanocrystals. ( $a$ and b) Effect of the reaction temperature on PL. (c and d) Effect of residence time on PL. All reactions were performed with $R_{1}=$ 1.8, $R_{2}=3.2, R_{3}=14 \%$, and $R_{4}=10 \%$. To index the green and red color, peaks at $543 \mathrm{~nm}$ and $652 \mathrm{~nm}$ were chosen, respectively. the current reactor set-up, since temperatures higher than this led to gas evolution from the reaction solution (the b.p. of TEGDME is $275{ }^{\circ} \mathrm{C}$ ). For the reaction time study, the temperature was set at $230{ }^{\circ} \mathrm{C}$ (to ensure sufficient PL intensity, whilst prohibiting gas evolution).

In this work we sought to minimize the required reaction time in order to increase the experimental throughput and the rapidity of optimization. As shown in Fig. 4c and d, the PL of the formed UCNPs increases with the residence time, approaching a plateau by $c a$. 4 minutes. Further, it was imperative to prove that the PL spectral shape (i.e. color) did not change substantially at longer reaction times versus the short reaction time to be used in our reactor. To confirm this, we ran a further bulk experiment to demonstrate that the PL (judged by the RGB\%) was consistent between a 4 minute reaction at $230{ }^{\circ} \mathrm{C}$ (as used in the microfluidic reactor) and a longer 'standard' reaction (we chose 60 minutes and $270{ }^{\circ} \mathrm{C}$ as representative) in the flask, which would justify our premise of using the microfluidic reactor to tune emission color. Although the size and phase of the nanocrystals did not remain constant (Fig. S3a-e, $\mathrm{ESI} \dagger$ ), the RGB\% values were consistent (Fig. S3f and g, ESI $\dagger$ ). Therefore, we determined that a reaction of 4 minutes and temperature of $230{ }^{\circ} \mathrm{C}$ was sufficient for rapid color tuning of the UCNP emission in our microfluidic reactor.

In Table 1 we provide a direct comparison between the synthesis method developed herein and other approaches from the literature. Overall, we find a Na-rich and F-deficient reaction formulation (versus the stoichiometry of $\mathrm{NaYF}_{4}: \mathrm{Yb}, \mathrm{Er}$ ) benefits the PL intensity, while color can be adjusted by tuning the doping degree of $\mathrm{Yb}^{3+}$. This is an important observation, and worthy of further study in future work. Moreover, results highlight the powerful capabilities of high-throughput microfluidic screening platforms in understanding complex synthetic systems. Indeed, in an experiment with $N$ synthetic variables (multiple precursors ratios, residence time, temperature, etc.), each having $M$ levels (defining the selected range of each factor), the overall number of required experimental iterations scales as $M^{N}$. The time needed for these iterations is then given by $M^{N} \times t$, where $t$ is the time for one iteration. In the case of $\mathrm{NaYF}_{4}: \mathrm{Yb}, \mathrm{Er}$ UCNPs, a reaction time of at least 60 minutes is typical for one bulk synthesis. Thus, conducting comprehensive parameter space mapping using standard flask-based approaches would rapidly become unmanageable due to the excessive time required, whereas it remains easily accessible using our modified synthesis route and screening approach.

\subsection{Synthesis and optimization of $\mathrm{NaYF}_{4}: \mathrm{Yb}, \mathrm{Er}, \mathrm{Tm}$}

As has been shown, tuning of $R_{1}-R_{4}$, residence time and temperature for $\mathrm{NaYF}_{4}: \mathrm{Yb}$,Er enables reaction optimization with respect to PL intensities and color tunability between green and orange. However, to obtain white-light-emitting UCNPs, another lanthanide ion is required for blue emission, whilst $\mathrm{Er}^{3+}$ contributes to green and red emission. $\mathrm{Tm}^{3+}$ ions are the typical blue source in upconversion, and are commonly co-doped into $\mathrm{NaYF}_{4}$ with $\mathrm{Er}^{3+}$ ions. ${ }^{42}$ White light emission is achieved by optimization of RGB emission to a quite specific 
a

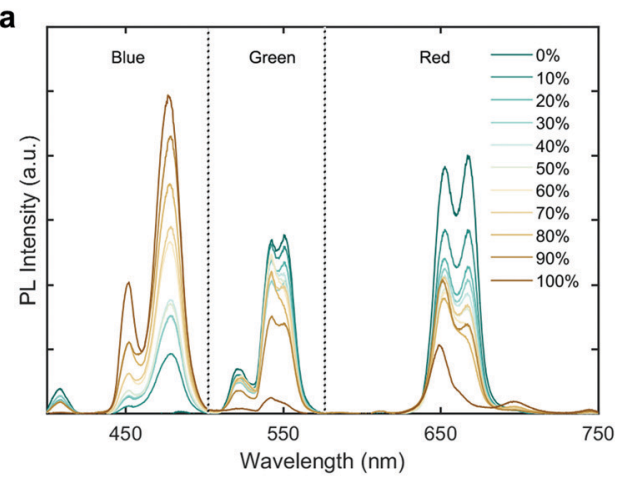

b

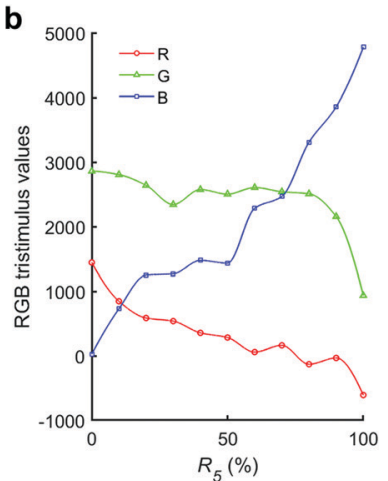

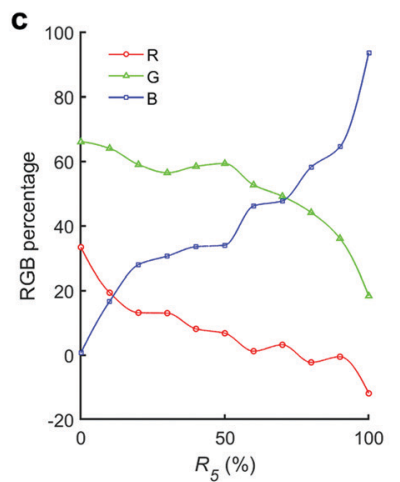
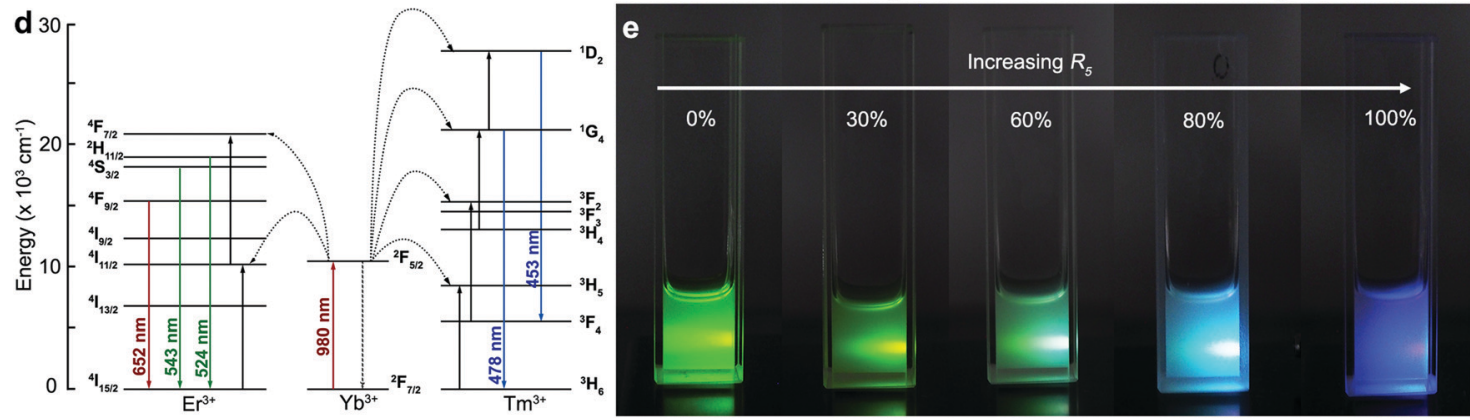

Fig. 5 Synthesis of color-tunable emission from NaYF 4 :Yb, Er,Tm UCNPs. (a) Effect of the Tm molar ratio $\left(R_{5}\right)$ on the PL of UCNPs. (b) The RGB tristimulus values of the UCNPs with different Tm molar ratio of $R_{5}$. (c) The RGB percentage of UCNPs with different Tm molar ratio of $R_{5}$. (d) Energy level scheme and color origins in upconversion. (e) Luminescence of nanocrystals in ethanol. Images were captured separately in the dark, with laser excitation at $980 \mathrm{~nm}$ (power density of $4 \mathrm{~W} \mathrm{~cm}^{-2}$ ).

doping ratio. ${ }^{9}$ Accordingly, we introduced a new variable, $R_{5}$ $(\mathrm{Tm} /(\mathrm{Tm}+\mathrm{Er}))$ to extract the optimal $\mathrm{Tm}$ : Er ratio. Specifically, we used the previously optimized parameters for $\mathrm{NaYF}_{4}: \mathrm{Yb}, \mathrm{Er}$, except that $R_{1}$ was varied to control the red-green balance. Fig. 5a shows spectra originating from $\mathrm{NaYF}_{4}: \mathrm{Yb}, \mathrm{Er}, \mathrm{Tm}(20 \%$ $\mathrm{Yb}$ ) with different degrees of Tm doping $\left(R_{5}\right)$. The blue region (with peaks at $478 \mathrm{~nm}$ and $453 \mathrm{~nm}$ ) arises from $\mathrm{Tm}^{3+}$, while green (543 and $524 \mathrm{~nm}$ ) and red (652 $\mathrm{nm}$ ) emissions arise from the $\mathrm{Er}^{3+}$ doping (Fig. 5d). To better understand the color evolution, spectra were converted into RGB tristimulus values based on the CIE colour matching functions. ${ }^{41}$ As $R_{5}$ increases, the blue value exhibits a continuous increase (Fig. 5b), and the red value a continuous decrease, across the entire range. The value of green emission was not significantly affected before an $R_{5}$ of $c a .80 \%$, but experienced a sharp decrease with any further increase in $\mathrm{Tm}^{3+}$ ratio. The percentage of the red and green emission (Fig. 5c) decreased with increasing $R_{5}$ (decreasing $\mathrm{Er}^{3+}$ ). The percentage of blue color increased from 0 to $95 \%$, as $R_{5}$ increased from 0 to $100 \%$. As a result of the low percentage of red color across the whole range of $R_{5}$, UCNP emission gradually changed from green to cold white and to blue with the different degrees of doping, as shown in Fig. 5e. The cold white light emitting $\mathrm{NaYF}_{4}: \mathrm{Yb}, \mathrm{Er}, \mathrm{Tm}$ nanocrystals were obtained for $R_{5}$ values between 70 and $80 \%$. Fig. $5 \mathrm{~d}$ shows the energy level diagram of $\mathrm{Yb}^{3+}-\mathrm{Er}^{3+}-\mathrm{Tm}^{3+}$ tri-doped $\mathrm{NaYF}_{4}$ nanocrystals. The $\mathrm{Yb}^{3+}$ is initially excited at $980 \mathrm{~nm}$ from its ground state ${ }^{2} \mathrm{~F}_{7 / 2}$ level to the ${ }^{2} \mathrm{~F}_{5 / 2}$ level, followed by energy transfer to $\mathrm{Er}^{3+}$ and $\mathrm{Tm}^{3+}$. The $\mathrm{Er}^{3+}$ then undergoes ${ }^{4} \mathrm{~S}_{3 / 2} \rightarrow{ }^{4} \mathrm{I}_{15 / 2}$ (543 nm, green), ${ }^{2} \mathrm{H}_{11 / 2} \rightarrow{ }^{4} \mathrm{I}_{15 / 2}\left(524 \mathrm{~nm}\right.$, green), and ${ }^{4} \mathrm{~F}_{9 / 2} \rightarrow{ }^{4} \mathrm{I}_{15 / 2}$
(652 nm, red) transitions. The $\mathrm{Tm}^{3+}$ undergoes ${ }^{1} \mathrm{G}_{4} \rightarrow{ }^{3} \mathrm{H}_{6}$ (478 nm, blue) and ${ }^{1} \mathrm{D}_{2} \rightarrow{ }^{3} \mathrm{~F}_{4}$ (453 nm, blue) transitions. In order to obtain white-light emission at a high excitation power, a high ratio of $R_{5}$ is required to ensure sufficient blue emission intensity. Fig. S4 (ESI $\dagger$ ) shows the CIE chromaticity diagram for $\mathrm{NaYF}_{4}: \mathrm{Yb}, \mathrm{Er}, \mathrm{Tm}$ UCNPs $(20 \% \mathrm{Yb})$ with varying values of $R_{5}$. The CIE chromaticity coordinates change from $x=0.3776, y=0.6091$ (green) to $x=0.2292, y=0.3585$ (cold white), ending at $x=0.1475$, $y=0.1121$ (blue), when increasing the doping ratio of $\mathrm{Tm}^{3+}$ from 0 to $100 \%$. With $20 \% \mathrm{Yb}^{3+}$, we achieve 'cold' white light rather than 'true' white due to a lack of red. However, by increasing the doping degree of $\mathrm{Yb}^{3+}$ to $60 \%$, we can increase the red light content (Fig. S5, ESI $\dagger$ ), thereby achieving true white light emission. The emission spectra of $\mathrm{NaYF}_{4}: \mathrm{Yb}, \mathrm{Er}, \mathrm{Tm}$ nanocrystals with $60 \% \mathrm{Yb}$ and variable Tm doping $\left(R_{5}\right)$ are shown in Fig. S5a (ESI $\dagger$ ). The red and green emission were similar across the entire range of $R_{5}$ (Fig. S5c and $\mathrm{d}$, ESI $\dagger$ ), which benefits targeting of true white light emission. The corresponding CIE chromaticity diagram (Fig. S5b, ESI $\dagger$ ) shows an $R_{5}$ of $60-70 \%$, yielding white light under $980 \mathrm{~nm}$ excitation (Movie S1, ESI $\dagger$ ). Overall, the emission colors of the products display a wide variation from orange to blue, and pass through the white emission zone.

The above results powerfully demonstrate the merits of rapid reaction tuning for $\mathrm{NaYF}_{4}: \mathrm{Yb}, \mathrm{Er}, \mathrm{Tm}$ UCNPs in obtaining precise multicolor emission in the visible region when exited by a single wavelength light source. Fig. 6a reports that the emission color of $\mathrm{NaYF}_{4}: \mathrm{Yb}, \mathrm{Er}, \mathrm{Tm}$ can be adjusted in two dimensions, by varying the $\mathrm{Yb}$ percentage to tune the greenred ratio, and varying the Tm percentage to tune the green-blue 

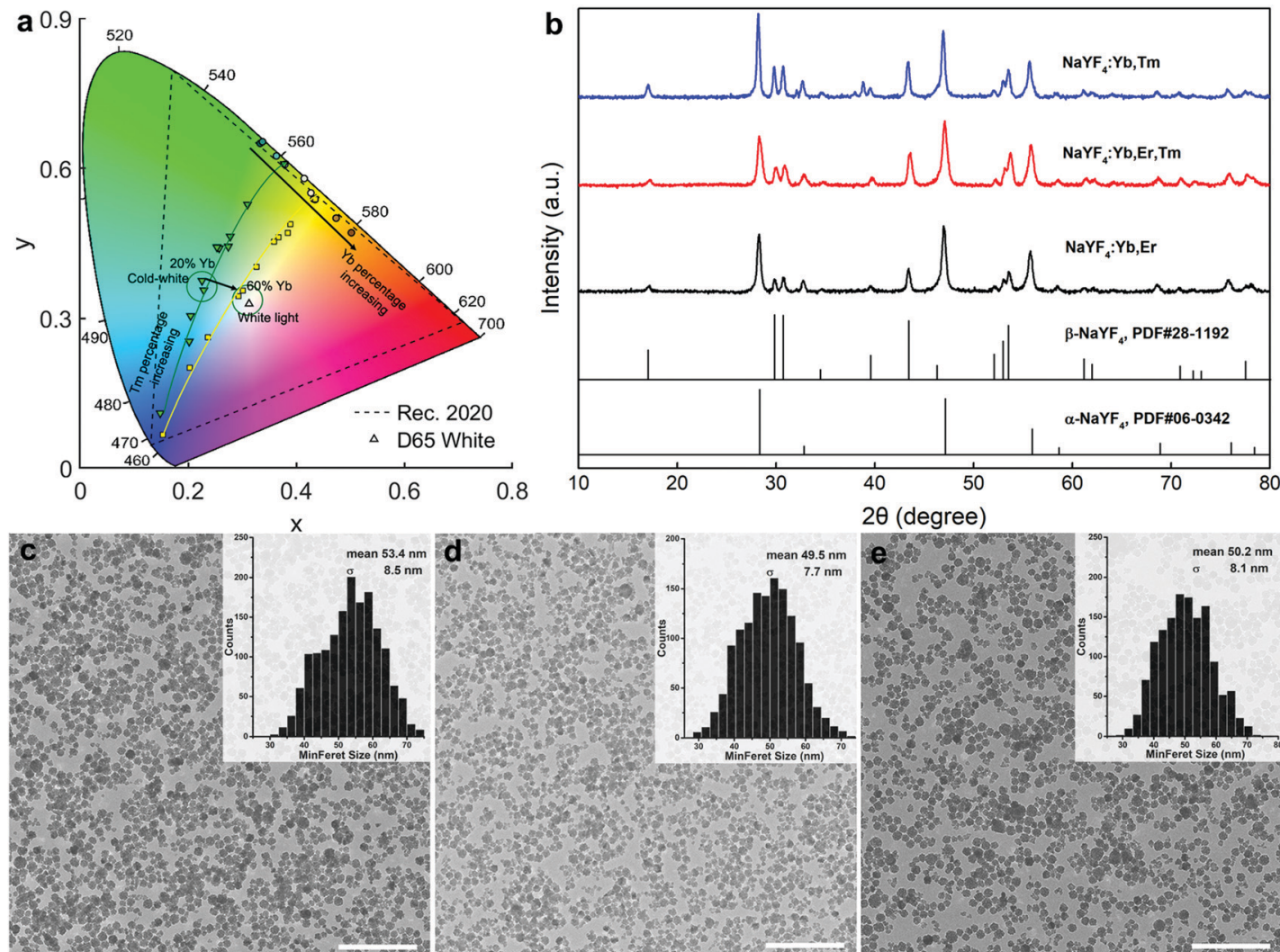

Fig. 6 (a) Plot of CIE1931 color coordinates of the NaYF 4 YY, Er,Tm UCNPs with different value of $R_{5}$ at $Y b$ doping degree at $20 \%$ and $60 \%$. (b) XRD patterns of $\mathrm{NaYF}_{4}: \mathrm{Yb}, \mathrm{Er}$, Tm UCNPs with $R_{5}$ at $0 \%$ (black), $70 \%$ (red), and $100 \%$ (blue). (c-e) TEM images of the nanocrystals with $R_{5}$ at $0 \%, 70 \%$, and $100 \%$. Scale bar is $500 \mathrm{~nm}$.

ratio. Fig. $6 \mathrm{~b}$ shows the XRD pattern of three samples, with $R_{5}$ equal to 0,70 and $100 \%$, yielding $\mathrm{NaYF}_{4}: \mathrm{Yb}, \mathrm{Tm}, \mathrm{NaYF}_{4}: \mathrm{Yb}, \mathrm{Er}, \mathrm{Tm}$, and $\mathrm{NaYF}_{4}: \mathrm{Yb}$,Er, respectively. All three samples exhibit peaks from both the cubic $(\alpha-)$ and hexagonal ( $\beta$-) phases. The diffraction peaks from the $\beta$-phase of the $0 \%$ sample $\left(\mathrm{NaYF}_{4}: \mathrm{Yb}, \mathrm{Er}\right)$ were weaker than those for the $70 \%\left(\mathrm{NaYF}_{4}: \mathrm{Yb}, \mathrm{Er}, \mathrm{Tm}\right)$ or $100 \%$ $\left(\mathrm{NaYF}_{4}: \mathrm{Yb}, \mathrm{Tm}\right)$ samples. This implies that the $\beta$-phase ratio in the absence of Tm is less than in the Tm-doped UCNPs. TEM images (Fig. S6, ESI $\dagger$ ) show that 'popcorn-like' $\mathrm{NaYF}_{4}: \mathrm{Yb}, \mathrm{Er}$ nanocrystals were obtained. The sizes of the nanocrystals (Fig. 6c-e) did not significantly vary as a function of Tm doping. The $R_{5}$ at 0,70 and $100 \%$ samples exhibited average diameters of $53.4 \pm 8.5,49.5 \pm 7.7$, and $50.2 \pm 8 \mathrm{~nm}$, respectively. Therefore, the size of the $\mathrm{NaYF}_{4}: \mathrm{Yb}, \mathrm{Er}, \mathrm{Tm}$ UCNPs seems to primarily depend on the $\mathrm{NaYF}_{4}: \mathrm{Yb}$ matrix rather than the doping degree of the activators.

\subsection{Application of $\mathrm{NaYF}_{4}: \mathrm{Yb}, \mathrm{Er}, \mathrm{Tm}$ nanocrystals}

To demonstrate the potential of using our color-tuned UCNPs in a thin film state, we fabricated a simple anti-counterfeit device. Here, a logo appears homogeneous under ambient light, but colored letters are revealed under infrared excitation. We recreated the ETH logo in a PDMS-based microdevice, which was fabricated via standard soft lithography. Subsequently, $\mathrm{NaYF}_{4}: \mathrm{Yb}, \mathrm{Er}, \mathrm{Tm}(20 \% \mathrm{Yb})$ UCNPs in ethanol (with $R_{5}$ at 0,70 and $100 \%$, representing three different emission colour profiles, see Fig. S7, ESI $\dagger$ ) were injected into the three character chambers separately, and dried by solvent evaporation. A smartphone camera was then used to capture images of the device through a simple lens-filter combination (Fig. 7a). Without the $980 \mathrm{~nm}$ excitation, all three characters appear identical (Fig. 7b). Once excited, the three different colors (green, cold white, and blue in Fig. 7c), can clearly be observed and imaged with the smartphone camera.
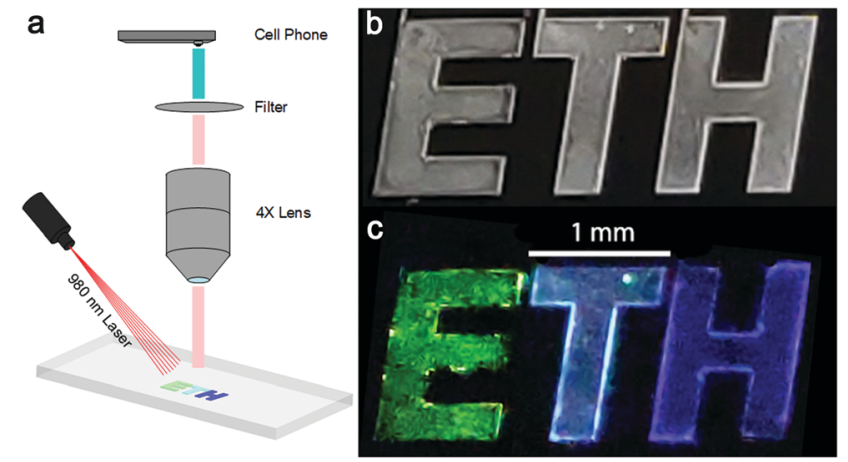

Fig. 7 Color-tunable $\mathrm{NaYF}_{4}$ :Yb,Er,Tm nanocrystals applied for anticountering. (a) Concept for anti-counterfeiting with imaging by a smartphone camera. (b) ETH logo captured without excitation. (c) Three different colors emerging under the $980 \mathrm{~nm}$ excitation. 


\section{Conclusions}

In summary, we have developed an entirely new synthesis strategy, in term of reagents, solvent and ligands, for the preparation and facile color tuning of $\mathrm{NaYF}_{4}: \mathrm{Yb}, \mathrm{Er}, \mathrm{Tm}$ UCNPs in a high-throughput manner. By taking advantage of the time and materials efficiency associated with droplet-based microfluidic reactors, compositional element ratios $\left(R_{1}\right.$ to $\left.R_{5}\right)$, as well as the residence time and reaction temperature could be optimized via inline PL analysis. Using a guided optimization of reaction parameters, color-tunable UCNPs have been synthesized by changing the doping degree of the $\mathrm{Yb}$ sensitizer (allowing controlled green-orange tuning) and the ratio between activators Tm-Er (achieving controlled green-blue tuning). With this two dimensional tuning, single white-light emitting $\mathrm{NaYF}_{4}: \mathrm{Yb}$,Er,Tm nanocrystals have been synthesized. As evidenced by the fabrication and testing of an anti-counterfeit device, the formed nanocrystals still exhibit excellent photoluminescence in the dried state. Finally, with this newly developed synthesis strategy, we have demonstrated great potential for fast screening of other host matrices, and coordinating ligands, as well as core-shell structures for various UCNP applications. Future work will focus on modifying the reactor to tolerate higher temperatures, testing solvents with higher boiling points, and exploring a wider range of precursors to measure their effects on particle properties.

\section{Author contributions}

Shangkun Li: conceptualization, investigation (all experiments), visualization, writing - original draft. Yingchao Meng: investigation (anticounterfeit device). Yujia Guo: investigation (COMSOL modelling). Tian Liu: investigation (XRD). Stavros Stavrakis: supervision, methodology, writing - review \& editing. Philip D. Howes: conceptualization, supervision, writing - original draft, review \& editing. Andrew J. deMello: funding acquisition, project administration, supervision, writing - review \& editing.

\section{Conflicts of interest}

The authors have no conflicts of interest to report.

\section{Acknowledgements}

The authors acknowledge partial financial support from ETH Zürich. S. L. acknowledges support from the China Scholarship Council (CSC), Grant \#201608140097. S. L. would like to thank Léonard Bezinge for assistance in CIE plotting. P. D. H. and S. S. would like to thank the Swiss National Science Foundation (SNSF) for support via a Spark Grant (Project No. CRSK2_190750). The authors are grateful to the ScopeM center at ETH for technical assistance in TEM imaging.

\section{References}

1 Z. Chen, C. L. Ho, L. Wang and W. Y. Wong, SingleMolecular White-Light Emitters and Their Potential WOLED Applications, Adv. Mater., 2020, 32, 1903269.

2 P. He, Y. Shi, T. Meng, T. Yuan, Y. Li, X. Li, Y. Zhang, L. Fan and S. Yang, Recent advances in white light-emitting diodes of carbon quantum dots, Nanoscale, 2020, 12, 4826-4832.

$3 \mathrm{H}$. Su, Y. Chen and K. Wong, Recent Progress in White Light-Emitting Electrochemical Cells, Adv. Funct. Mater., 2019, 1906898.

4 S. Sivakumar, F. C. J. M. Van Veggel and M. Raudsepp, Bright white light through up-conversion of a single NIR source from sol-gel-derived thin film made with $\mathrm{Ln}^{3+}$-doped $\mathrm{LaF}_{3}$ nanoparticles, J. Am. Chem. Soc., 2005, 127, 12464-12465.

5 E. Jang, S. Jun, H. Jang, J. Lim, B. Kim and Y. Kim, Whitelight-emitting diodes with quantum dot color converters for display backlights, Adv. Mater., 2010, 22, 3076-3080.

6 T. Higuchi, H. Nakanotani and C. Adachi, High-Efficiency White Organic Light-Emitting Diodes Based on a Blue Thermally Activated Delayed Fluorescent Emitter Combined with Green and Red Fluorescent Emitters, Adv. Mater., 2015, 27, 2019-2023.

7 K. T. Kamtekar, A. P. Monkman and M. R. Bryce, Recent advances in white organic light-emitting materials and devices (WOLEDS), Adv. Mater., 2010, 22, 572-582.

8 P. T. Furuta, L. Deng, S. Garon, M. E. Thompson and J. M. J. Fréchet, Platinum-functionalized random copolymers for use in solution-processible, efficient, near-white organic lightemitting diodes, J. Am. Chem. Soc., 2004, 126, 15388-15389.

9 C. Zhang, L. Yang, J. Zhao, B. Liu, M. Y. Han and Z. Zhang, White-Light Emission from an Integrated Upconversion Nanostructure: Toward Multicolor Displays Modulated by Laser Power, Angew. Chem., Int. Ed., 2015, 54, 11531-11535.

10 Z. He, W. Zhao, J. W. Y. Lam, Q. Peng, H. Ma, G. Liang, Z. Shuai and B. Z. Tang, White light emission from a single organic molecule with dual phosphorescence at room temperature, Nat. Commun., 2017, 8, 416.

11 S. Wen, J. Zhou, K. Zheng, A. Bednarkiewicz, X. Liu and D. Jin, Advances in highly doped upconversion nanoparticles, Nat. Commun., 2018, 9, 2415.

12 D. Sarkar, S. Ganguli, T. Samanta and V. Mahalingam, Design of Lanthanide-Doped Colloidal Nanocrystals: Applications as Phosphors, Sensors, and Photocatalysts, Langmuir, 2019, 35, 6211-6230.

13 G. Tessitore, G. A. Mandl, M. G. Brik, W. Park and J. A. Capobianco, Recent insights into upconverting nanoparticles: spectroscopy, modeling, and routes to improved luminescence, Nanoscale, 2019, 11, 12015-12029.

14 K. Zheng, K. Y. Loh, Y. Wang, Q. Chen, J. Fan, T. Jung, S. H. Nam, Y. D. Suh and X. Liu, Recent advances in upconversion nanocrystals: Expanding the kaleidoscopic toolbox for emerging applications, Nano Today, 2019, 29, 100797.

15 R. D. Mehlenbacher, R. Kolbl, A. Lay and J. A. Dionne, Nanomaterials for in vivo imaging of mechanical forces and electrical fields, Nat. Rev. Mater., 2018, 3, 17080. 
16 Y. Wang, S. Song, S. Zhang and H. Zhang, Stimuliresponsive nanotheranostics based on lanthanide-doped upconversion nanoparticles for cancer imaging and therapy: current advances and future challenges, Nano Today, 2019, 25, 38-67.

17 J. Zhou, J. L. Leaño, Z. Liu, D. Jin, K.-L. Wong, R.-S. Liu and J.C. G. Bünzli, Impact of Lanthanide Nanomaterials on Photonic Devices and Smart Applications, Small, 2018, 14, 1801882.

18 C. D. S. Brites, S. Balabhadra and L. D. Carlos, LanthanideBased Thermometers: At the Cutting-Edge of Luminescence Thermometry, Adv. Opt. Mater., 2019, 7, 1801239.

19 Z. Li, H. Yuan, W. Yuan, Q. Su and F. Li, Upconversion nanoprobes for biodetections, Coord. Chem. Rev., 2018, 354, 155-168.

20 Y. Hu, Q. Shao, X. Deng, D. Song, S. Han, Y. Dong and J. Jiang, Thermally induced multicolor emissions of upconversion hybrids with large color shifts for anticounterfeiting applications, J. Mater. Chem. C, 2019, 7, 11770-11775.

21 S. Wang, J. Chen, J. Lin, C. Yang, F. Huang and D. Chen, Nanocrystallization of lanthanide-doped KLu2F7-KYb2F7 solid-solutions in aluminosilicate glass for upconverted solid-state-lighting and photothermal anti-counterfeiting, J. Mater. Chem. C, 2019, 7, 14571-14580.

22 A. J. Evangelista, M. Ivanchenko, A. F. Myers, L. N. McAnulty, G. K. M. Payne and H. Jing, Multi-shelled upconversion nanostructures with enhanced photoluminescence intensity via successive epitaxial layer-by-layer formation (SELF) strategy for high-level anticounterfeiting, J. Mater. Chem. C, 2020, 8, 5692-5703.

$23 \mathrm{~J}$. Wang and P. A. Tanner, Upconversion for white light generation by a single compound, J. Am. Chem. Soc., 2010, 132, 947-949.

24 T. Wang, H. Yu, C. K. Siu, J. Qiu, X. Xu and S. F. Yu, WhiteLight Whispering-Gallery-Mode Lasing from LanthanideDoped Upconversion $\mathrm{NaYF}_{4}$ Hexagonal Microrods, ACS Photonics, 2017, 4, 1539-1543.

25 K. Lingeshwar Reddy, R. Balaji, A. Kumar and V. Krishnan, Lanthanide Doped Near Infrared Active Upconversion Nanophosphors: Fundamental Concepts, Synthesis Strategies, and Technological Applications, Small, 2018, 14, 1801304.

26 Y. Sun, Y. Chen, L. Tian, Y. Yu, X. Kong, J. Zhao and H. Zhang, Controlled synthesis and morphology dependent upconversion luminescence of $\mathrm{NaYF}_{4}: \mathrm{Yb}$, Er nanocrystals, Nanotechnology, 2007, 18, 275609.

$27 \mathrm{Z}$. Li and Y. Zhang, An efficient and user-friendly method for the synthesis of hexagonal-phase $\mathrm{NaYF}_{4}: \mathrm{Yb}, \mathrm{Er} / \mathrm{Tm}$ nanocrystals with controllable shape and upconversion fluorescence, Nanotechnology, 2008, 19, 345606.

28 H. X. Mai, Y. W. Zhang, R. Si, Z. G. Yan, L. D. Sun, L. P. You and C. H. Yan, High-quality sodium rare-earth fluoride nanocrystals: Controlled synthesis and optical properties, J. Am. Chem. Soc., 2006, 128, 6426-6436.

29 B. K. H. Yen, N. E. Stott, K. F. Jensen and M. G. Bawendi, A Continuous-Flow Microcapillary Reactor for the Preparation of a Size Series of CdSe Nanocrystals, Adv. Mater., 2003, 15, 1858-1862.
30 H. Nakamura, Y. Yamaguchi, M. Miyazaki, H. Maeda, M. Uehara and P. Mulvaney, Preparation of CdSe nanocrystals in a micro-flow-reactor, Chem. Commun., 2002, 2844-2845.

31 J. B. Edel, R. Fortt, J. C. DeMello and A. J. DeMello, Microfluidic routes to the controlled production of nanoparticles, Chem. Commun., 2002, 1136-1137.

32 H. Liu, O. Jakobsson, C. T. Xu, H. Xie, T. Laurell and S. Andersson-Engels, in Colloidal Quantum Dots/Nanocrystals for Biomedical Applications VI, ed. W. J. Parak, K. Yamamoto and M. Osinski, SPIE, 2011, vol. 7909, p. 790917.

33 D. Liu, Y. Jing, K. Wang, Y. Wang and G. Luo, Reaction study of $\alpha$-phase $\mathrm{NaYF}_{4}: \mathrm{Yb}, \mathrm{Er}$ generation via a tubular microreactor: Discovery of an efficient synthesis strategy, Nanoscale, 2019, 11, 8363-8371.

34 J. Sui, J. Yan, K. Wang and G. Luo, Efficient synthesis of lithium rare-earth tetrafluoride nanocrystals via a continuous flow method, Nano Res., 2020, 13, 2837-2846.

35 I. Lignos, S. Stavrakis, G. Nedelcu, L. Protesescu, A. J. DeMello and M. V. Kovalenko, Synthesis of Cesium Lead Halide Perovskite Nanocrystals in a Droplet-Based Microfluidic Platform: Fast Parametric Space Mapping, Nano Lett., 2016, 16, 1869-1877.

36 J. Nette, P. D. Howes and A. J. DeMello, Microfluidic Synthesis of Luminescent and Plasmonic Nanoparticles: Fast, Efficient, and Data-Rich, Adv. Mater. Technol., 2020, 2000060.

37 S. Li, R. W. Baker, I. Lignos, Z. Yang, S. Stavrakis, P. D. Howes and A. J. DeMello, Automated microfluidic screening of ligand interactions during the synthesis of cesium lead bromide nanocrystals, Mol. Syst. Des. Eng., 2020, 5, 1118-1130.

38 Y. Ding, P. D. Howes and A. J. Demello, Recent Advances in Droplet Microfluidics, Anal. Chem., 2020, 92, 132-149.

39 R. A. Janjua, C. Gao, R. Dai, Z. Sui, M. A. Ahmad Raja, Z. Wang, X. Zhen and Z. Zhang, $\mathrm{Na}^{+}$-Driven Nucleation of $\mathrm{NaYF}_{4}: \mathrm{Yb}, \mathrm{Er}$ Nanocrystals and Effect of Temperature on Their Structural Transformations and Luminescent Properties, J. Phys. Chem. C, 2018, 122, 23242-23250.

40 F. Vetrone, J. C. Boyer, J. A. Capobianco, A. Speghini and M. Bettinelli, Significance of $\mathrm{Yb}^{3+}$ concentration on the upconversion mechanisms in codoped $\mathrm{Y}_{2} \mathrm{O}_{3}: \mathrm{Er}^{3+}, \mathrm{Yb}^{3+}$ nanocrystals, J. Appl. Phys., 2004, 96, 661-667.

41 H. S. Fairman, M. H. Brill and H. Hemmendinger, How the CIE 1931 color-matching functions were derived from Wright-Guild data, Color Res. Appl., 1997, 22, 11-23.

42 G. S. Yi and G. M. Chow, Synthesis of hexagonal-phase $\mathrm{NaYF}_{4}: \mathrm{Yb}, \mathrm{Er}$ and $\mathrm{NaYF}_{4}: \mathrm{Yb}, \mathrm{Tm}$ nanocrystals with efficient up-conversion fluorescence, Adv. Funct. Mater., 2006, 16, 2324-2329.

43 Y. Wei, F. Lu, X. Zhang and D. Chen, Synthesis of oildispersible hexagonal-phase and hexagonal-shaped NaY$\mathrm{F}_{4}: Y b$, Er nanoplates, Chem. Mater., 2006, 18, 5733-5737.

44 H. Schäfer, P. Ptacek, H. Eickmeier and M. Haase, Synthesis of hexagonal $\mathrm{Yb}^{3+}, \mathrm{Er}^{3+}$-doped $\mathrm{NaYF}_{4}$ nanocrystals at low temperature, Adv. Funct. Mater., 2009, 19, 3091-3097. 